\title{
Quantitative Study on Individual Representations in Senegalese Patients Living with HIV (PLHIV)
}

\author{
Racky Wade-Kane ${ }^{*}$, Momar Camara1, El Hadji Makhtar Ba1 , Idrissa Ba ${ }^{2}$, Sokhna Seck ${ }^{1}$, \\ Abou Sy' ${ }^{1}$ Ibrahima Ndiaye ${ }^{2}$, Oumar Mamadou Samba ${ }^{2}$, Ndéye Dialé Ndiaye Ndongo', \\ Papa Lamine Faye ${ }^{1}$, Aida Sylla ${ }^{1}$, Mamadou Habib Thiam ${ }^{1}$ \\ ${ }^{1}$ Department of Psychiatry and Medical Psychology, National University Hospital Center of Fann, Dakar, Senegal \\ ${ }^{2}$ Center for the Integrated Support of Addictions in Dakar (CEPIAD), National University Hospital Center of Fann, Dakar, \\ Senegal \\ Email: ^racky.wade@hotmail.com
}

How to cite this paper: Wade-Kane, R., Camara, M., Ba, El H.M., Ba, I., Seck, S., Sy, A., Ndiaye, I., Samba, O.M., Ndongo, N.D.N., Faye, P.L., Sylla, A. and Thiam, M.H. (2022) Quantitative Study on Individual Representations in Senegalese Patients Living with HIV (PLHIV). Open Journal of Psychiatry, 12, 23-36.

https://doi.org/10.4236/ojpsych.2022.121003

Received: November 5, 2021

Accepted: November 29, 2021

Published: December 2, 2021

Copyright $\odot 2022$ by author(s) and Scientific Research Publishing Inc. This work is licensed under the Creative Commons Attribution International License (CC BY 4.0).

http://creativecommons.org/licenses/by/4.0/

\begin{abstract}
Context: HIV infection is an infectious, viral, chronic disease described as a public health problem. It has an impact on bio-psycho-social well-being. Thus, the objective of our study is to decrypt the representations of HIV infection in our patients. Methodology: 31 consecutively recruited HIV-infected patients participated in our study. The completed questionnaire included socio-demographic characteristics, association membership, psycho-social aspects of HIV infection, mode of transmission and adherence to antiretroviral treatment. Secondly, we proceeded to an assessment of the psychological state of the patient. Results: Almost 77\% of HIV patients do not date peers in an association. At the time of the study, more than half of patients or 19/31 with HIV had a positive image of the disease. Before the diagnosis of the disease, more than $2 / 3$ of patients with HIV, i.e. 25/31, have a negative image of the disease. The 31 PLHIV have been aware of their positive HIV status for an average of 11 years with extremes of 2 years and 21 years. Almost 21/31 of PLHIV have shared their positive serological status. The main barrier in patient communication was the risk of disclosure of HIV status. Most of the patients (45.2\%) thought of death when they announced their positive HIV status, and $19.4 \%$ thought of their children. In $80.6 \%$ of cases, relationships with family, friends and neighbors were normal and remained unchanged for $74 \%$ of patients. The patients thought they had been infected through sexual intercourse in $84 \%$ of cases. Our study showed that 6 of the 31 PLHIV experienced stigma. Among these 6 patients, 3 had psychological repercussions and a voluntary interruption of treatment. Conclusion: The representations are dynamic. In Senegal, the eradication of discrimination among people living with HIV must imperatively involve a reconstruction of individual, col-
\end{abstract}


lective and social representations.

\section{Keywords}

HIV, Representations, Stigma, Senegal

\section{Introduction}

HIV infection is an infectious, viral, chronic disease caused by human immunodeficiency viruses (HIV-1 and HIV-2) [1]. It has been described as a public health problem from its origins to the present day. It is characterized by a decrease in the body's defenses which, when significant, leads to the onset of many opportunistic diseases. HIV infection also impacts psychosocial well-being. Thus, taking into account the somatic and psychic fields of this condition is essential for a comprehensive understanding of the person [2]. Collective or individual representations of HIV infection correspond to the perception, meaning or mental image that the patient (or the society in which he lives) has of this disease. Individual representations are those that the individual constructs through interaction with his environment. They constitute a coherent and personal whole and serve to organize its action. Collective representations refer to those shared by a social group in terms of content primarily. They serve to define common ways of thinking (around norms, myths, objectives) which regulate and legitimize behavior within the group. The notion of collective representations insists on their specificity for the group which develops and shares them. Social representation designates more the representations studied in their dynamics, their development, their evolutions than in their content. These social representations integrate collective and individual aspects. For Moscovici (1989), to represent a thing, a state is in fact not simply duplicating it, repeating it or reproducing it, it is reconstituting it, retouching it, changing its text [3] [4]. The study of social representations better corresponds to the needs of modern, changing and communicating society.

The representation tells us the common code of a group, its culture, but also everything that crosses this group: its group imagination. This partly explains its decisive impact on the behavior of the general population. We did not find in the literature any studies done in Senegal specifically on the types of representations found in patients living with HIV (PLHIV). Thus, the objective of our study is to decipher the representations of the HIV infection of our patients through their speech and the form they will have filled out.

\section{Methodology}

\subsection{Patients and Method}

\subsubsection{Type and Period of Study}

Our study was quantitative longitudinal for descriptive and analytical purposes. It took place over a continuous period from April 21 to June 12, 2015. 


\subsubsection{Study Population}

Our study population consisted of 31 patients infected with HIV. The sample was formed through consecutive recruitment based on the daily consultation list of PLHIV followed at the outpatient treatment center (OTC) and meeting the selection criteria.

- Inclusion criteria

The following patients were included in our study:

- Of Senegalese cultural origin;

- Diagnosed with HIV and informed of their status;

- Aged 18 or over;

- Having freely consented to participate in our study after being informed.

- Criteria for non-inclusion

Patients were not included in our study:

- Strangers to Senegalese culture;

- Under the age of 18 ;

- Not infected with HIV;

- Not followed up at OTS;

- Not yet informed of their serological status;

- Not consenting to participate in our study.

- Exclusion criteria

Were excluded, patients:

- Whose questionnaires are incomplete;

- No longer consenting to participate in our study.

\subsubsection{Collection Tools and Methods}

After free and informed consent in the consultation room, we conducted a semi-structured interview based on a questionnaire tested and validated by our supervisors. The questionnaire is made up of different parts comprising each of the variables and their modalities. It's about:

- Socio-demographic characteristics (age, sex, address, profession, level of education, marital status, religion, cultural affiliation);

- Membership of an association;

- Psycho-social aspects of HIV infection:

- Individual representations with questions: What is your current perception of HIV infection? Did you have any other meaning of HIV infection before you found out that you were positive?;

- Stigmatization;

- Discrimination;

- Disclosure of positive HIV status to others.

- The mode of transmission;

- Adherence to ARV treatment.

After completing the questionnaire we proceeded to an assessment of the patient's psychological state. 


\subsubsection{Data Entry and Use}

With the help of a statistician we made an input mask. These data were extracted and then analyzed using the Microsoft Office Excel 2007 program.

\subsection{Contact}

At the very beginning of our work, we asked for a meeting with the President and 4 other members of the National Network of PvVIH (RNP), in order to get their impression on the theme of our study. During this meeting, the members present uniformly suggested that we propose questionnaires that were not exhaustive and easy to complete. They also expressed their willingness to have an overview on the questionnaire before starting the survey.

\subsection{Constraints and Limits}

We were faced with a problem of time to complete the interviews. Some patients wanted to continue the interview more to talk about their experiences and the difficulties encountered on a daily basis. Others urged us to have the integrity of their speech written on the questionnaire so that their concerns could be taken into account by the authorities and by all other actors.

\subsection{Ethical, Legal and Administrative Considerations}

Our study protocol was approved by the Chief Medical Officer responsible for OTC.

All information has been collected taking into account compliance with the rules of medical ethics, namely:

- Anonymity: patient identification is summarized by a number assigned to him and which appears on the questionnaire;

- Free and informed consent: the patient has received an information sheet on the work we do. This sheet has been read and explained by the case worker in a language he understands well.

\section{Results}

\subsection{Distribution of 31 PLHIV by Sex}

The majority of patients surveyed are women (54.8\%) [Figure 1].

\subsection{Distribution of 31 PLHIV According to Marital Status}

According to marital status regarding marital status, $29 \%$ of patients were widowers [Figure 2].

\subsection{Distribution of 31 PvVIH According to Membership of an Association}

Almost 24 out of $31(77.4 \%)$ HIV patients do not socialize with peers in an association. Those who associate with peers are generally women (5 out of 7 (71\%) patients who associate with peers are women) [Figure 3]. 


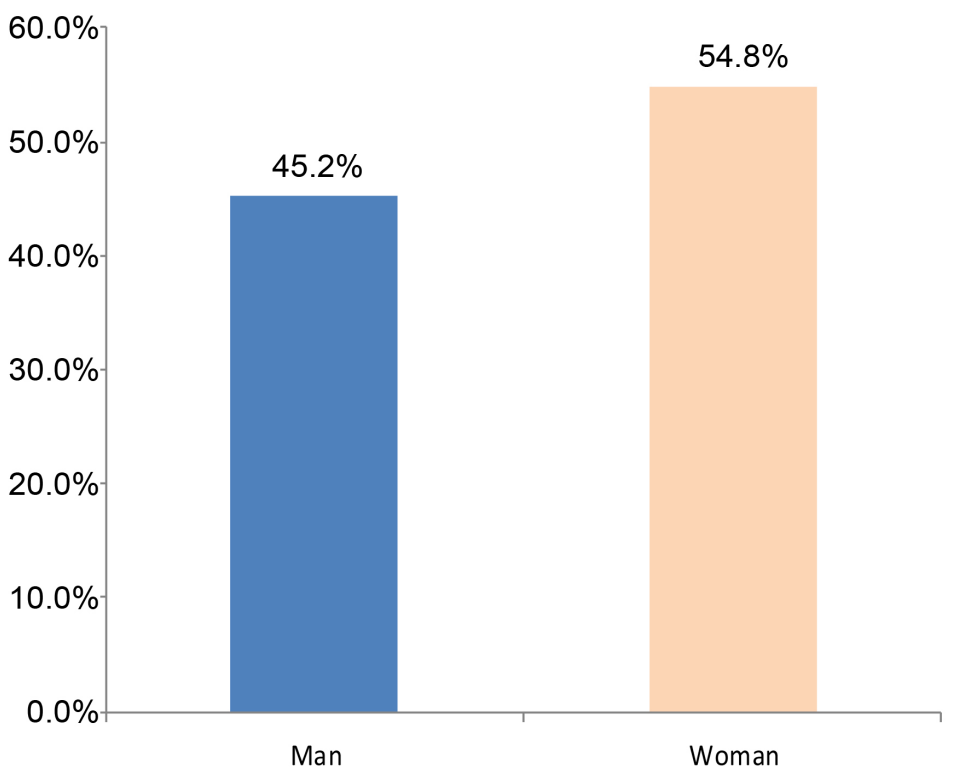

Figure 1. Distribution of 31 PLHIV by sex.

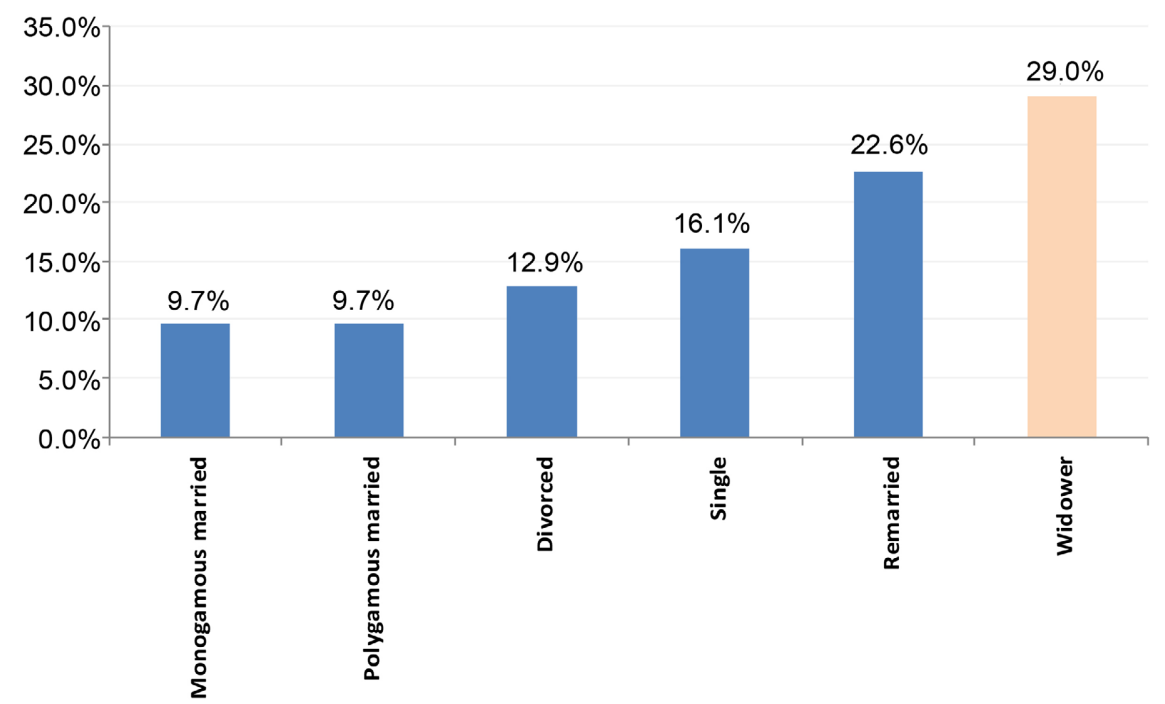

Figure 2. Distribution of 31 PLHIV according to marital status.

\subsection{Perception of HIV Infection by 31 PLHIV}

Are considered a positive representation:

- Disease like any other disease

- Illness caused by a virus

- Divine will

Are considered a negative representation:

- Bad disease

- Contagious illness

- Incurable disease

- Burden

- Distressing illness 
- AIDS

- Disease of infidels

- Non-existent disease

\subsubsection{Current Perception of HIV Infection}

More than half of patients with HIV, i.e. 19/31 (61.3\%) have a positive image of the disease. The $12 / 31$ or $38.7 \%$ of patients with HIV have a negative image (HIV perceived as a burden, a bad disease or a painful disease). This image reflects how they had HIV before knowing their positive status [Figure 4].

\subsubsection{Perception of HIV Infection before Diagnosis of HIV Infection}

More than $2 / 3$ of patients with HIV, i.e. $25 / 31$ (80.6\%), have a negative image of the disease. The most common perception of HIV infection is that which is described as a disease of infidels [Figure 5].

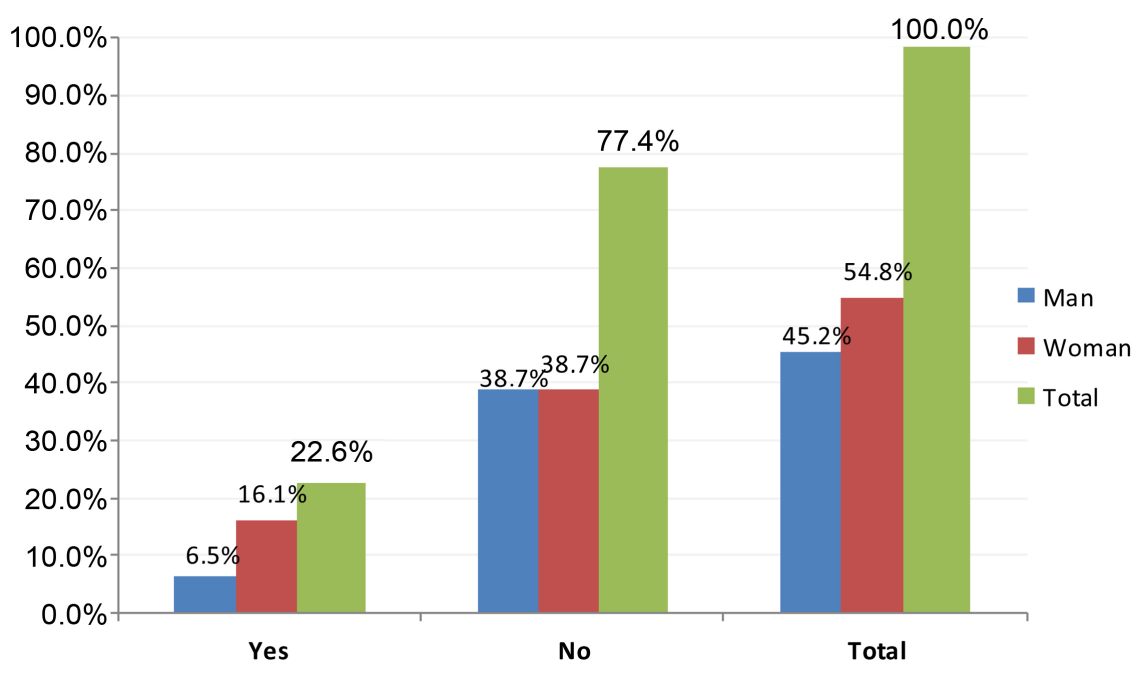

Figure 3. Distribution of $31 \mathrm{PvVIH}$ according to membership of an association.

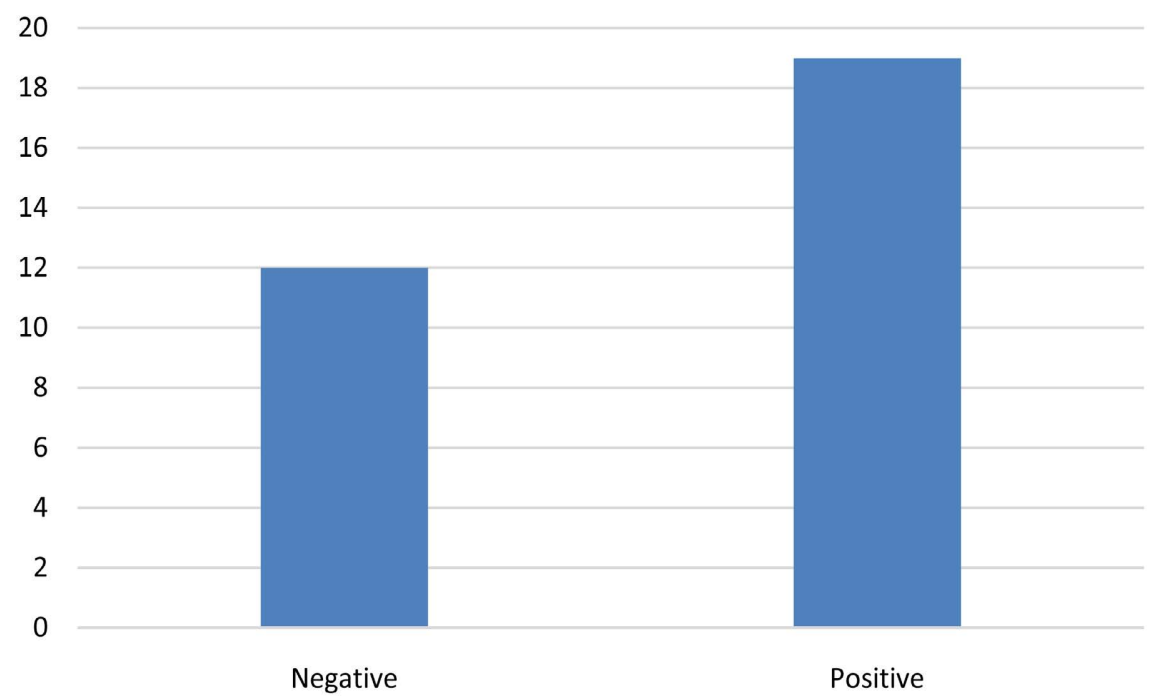

Figure 4. Distribution of the current perception of HIV infection among 31 PLHIV. 


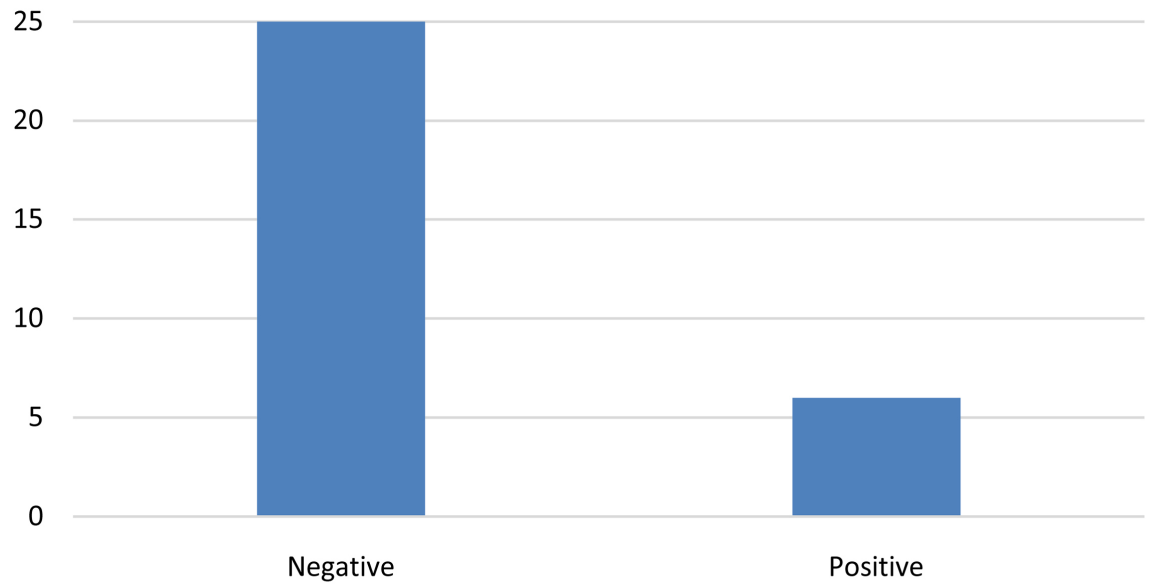

Figure 5. Distribution of the perception of HIV infection before the announcement of positive serological status among 31 PLHIV.

\subsection{Duration of Knowledge of Positive HIV Status}

The 31 PLHIV have been aware of their positive HIV status for an average of 11 years with extremes of 2 years and 21 years.

\subsection{Sharing Positive HIV Status}

Almost 2/3, or 21 out of 31, of PLHIV shared their positive serological status. Men shared most often with their spouse. As for the women, they preferred their parents. The main reason that led them to share their status is trust (10/21 of PLHIV).

The other 10 patients did not share their status. The first reason is fear of stigma (5/10) and the second is fear of disclosing their positive HIV status (3/10) [Table 1].

\subsection{Communication of Positive HIV Status}

21/31 of PLHIV communicate hardly their positive serological status [Figure 6].

\subsection{The Reasons for Difficult Communication of Positive HIV Status}

The main barrier in patient communication was the risk of disclosure of HIV status [Figure 7].

\subsection{What Did Patients Think during the Hours Following the Announcement of Their Positive HIV Status?}

Patients' thinking about announcing their positive HIV status differs from one individual to another depending on its severity. Most of the patients (45.2\%) thought of death when they announced their positive HIV status, and 19.4\% thought of their children. Some patients (9.7\%) thought of the risk of infection 
Table 1. Distribution according to confidant of the 21 PLHIV sharing their positive serological status.

\begin{tabular}{cccc}
\hline Confidant & Man & Woman & Total \\
\hline Friend & 2 & 0 & 2 \\
Spouse & 4 & 1 & 5 \\
Parent & 3 & 10 & 13 \\
Upper hierarchy & 1 & 0 & 1 \\
Total & 10 & 11 & 21 \\
\hline
\end{tabular}

25

20

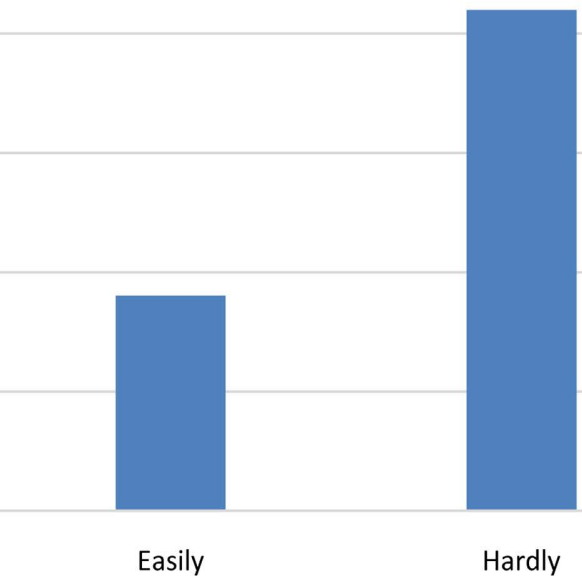

0

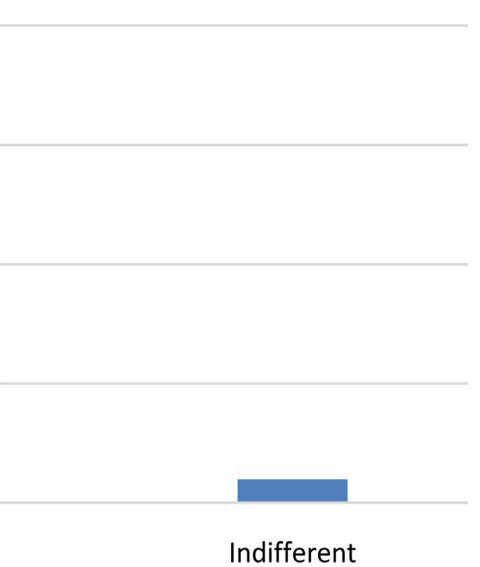

Figure 6. Distribution of the 31 PLHIV according to their facility of communicating their positive serological status.

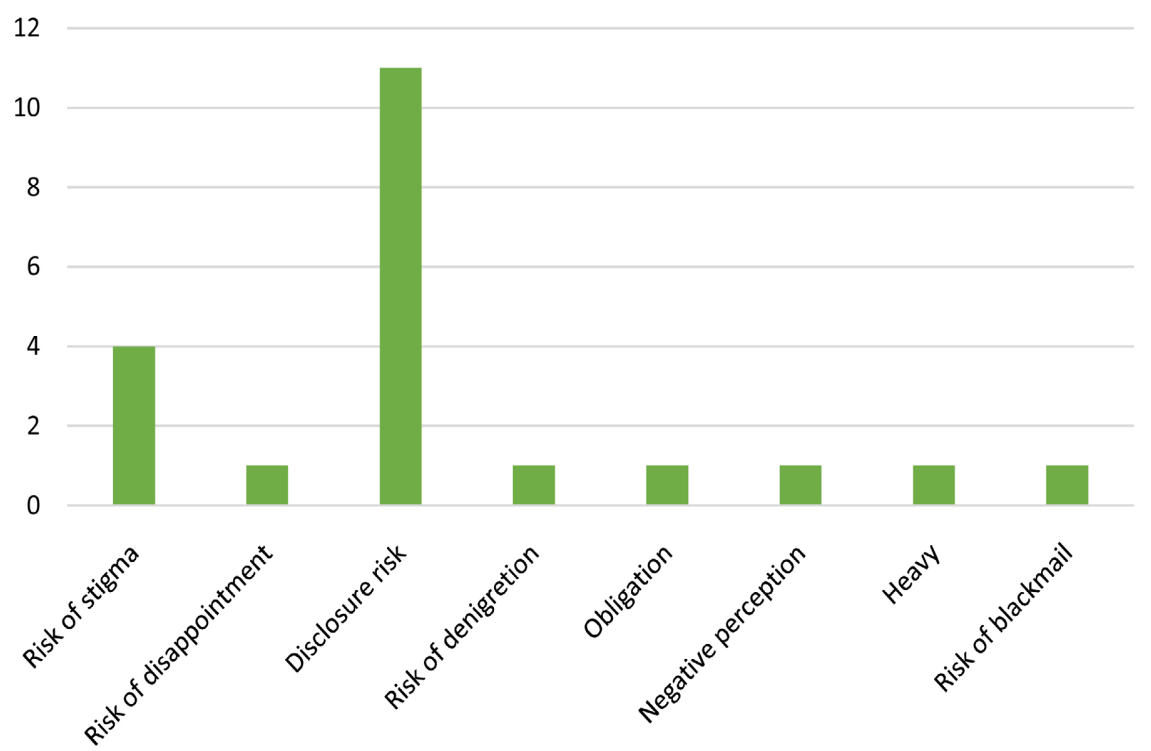

Figure 7. Distribution of 31PLHIV according to the reasons given for difficult communication of positive HIV status. 
while others thought of suicide (3.2\%). Others, on the other hand, have thought of a confidant.

\subsection{Relationship with Family Members, Neighbors and Friends}

In $80.6 \%$ of cases, relationships with family, friends and neighbors were normal [Figure 8].

\subsection{Evolution of Relationships with Family Members, Neighbors and Friends}

Relationships with family, friends and neighbors were remained unchanged for $74.2 \%$ of patients [Figure 9].

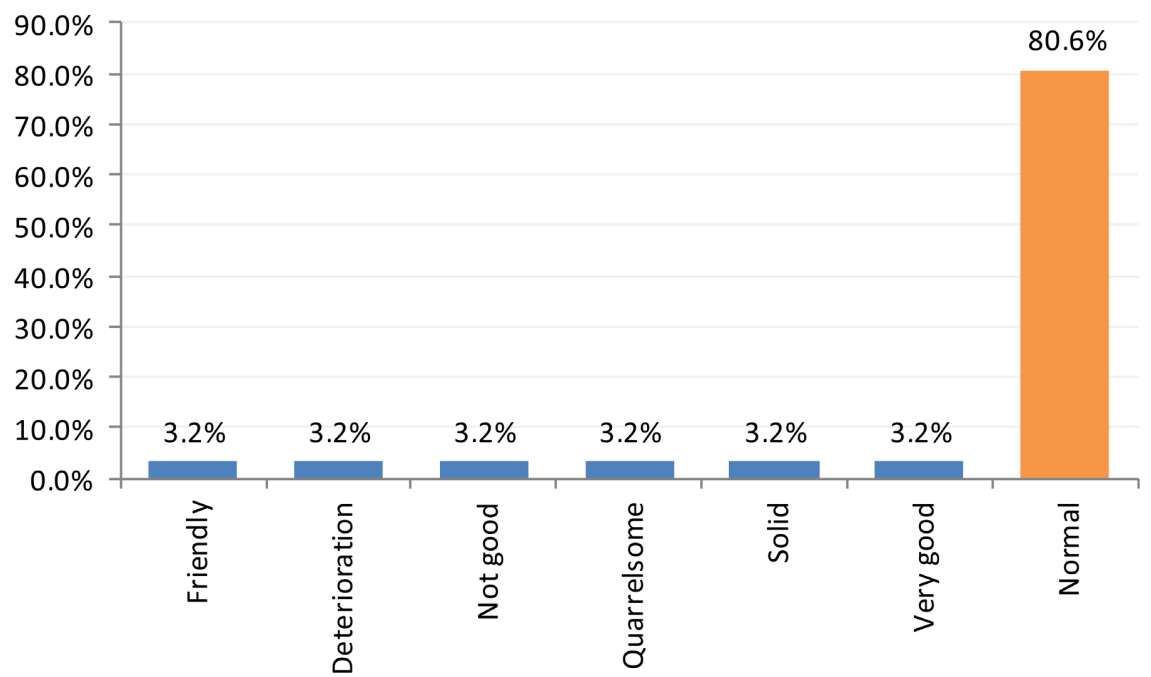

Figure 8. Distribution of the $31 \mathrm{PvVIH}$ according to the type of relationship with the entourage.

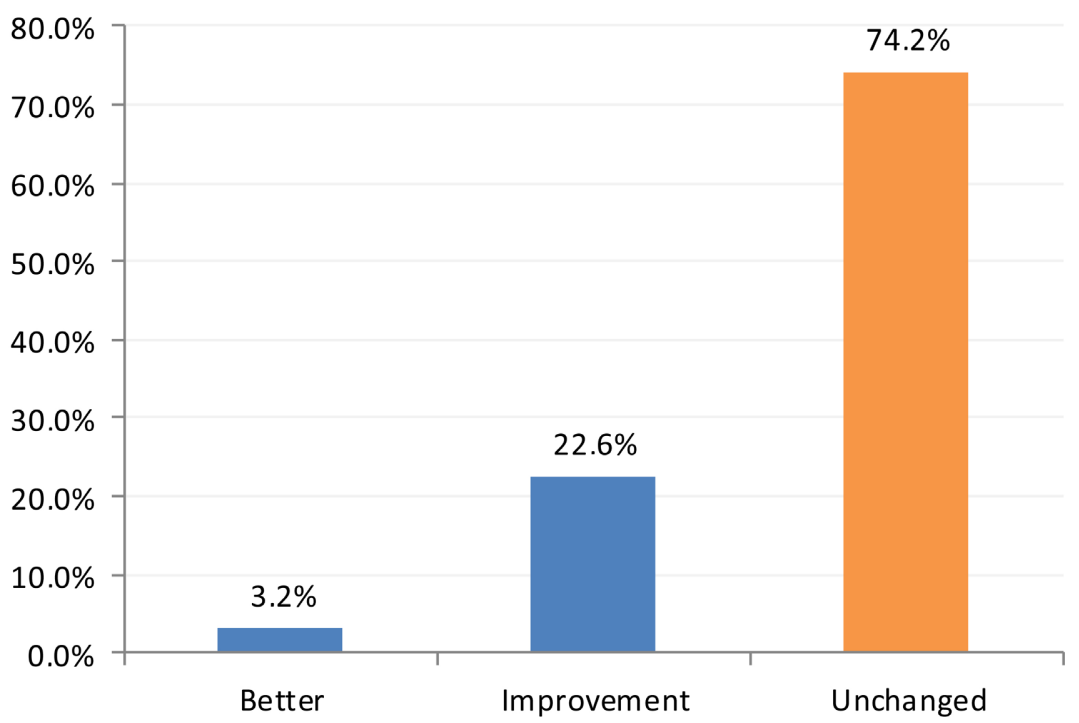

Figure 9. Distribution of 31 PLHIV according to the evolution of relationships with those around them. 


\subsection{Overview of Transmission Mode}

The patients thought they had been infected through sexual intercourse in $84 \%$ of cases [Figure 10].

\subsection{Stigma}

Our study showed that 6 of the 31 PLHIV were victims of stigma such as rejection, avoidance, denigration, even leading to relocations. Of these 6 patients, 3 had current psychological repercussions such as depression or anxiety; two others voluntarily interrupted their treatment.

\section{Discussion}

\subsection{The Representations}

To study a representation is to study both what people think of a particular object (content of the representation), but also how they think (socio-cognitive organizations that explain this content) and why they think it.

In general, HIV infection is currently perceived by patients as positive. We can consider these current perceptions as being individual representations of the HIV infection because Clenet (1998) qualifies these representations of "what a subject could internalize of a lived situation, of what for him makes sense and gives meaning to its actions" [3]. The PLHIV included in our study have known their HIV status for an average of 11 years. During these years, they are followed by the OTC which offers them a package of services including psycho-social monitoring, education and training. In addition to the various interactions with their social, physical and cultural environment, these PLHIV have built their individual representations. Ben (2005) concludes in his study that individual representations will serve as a framework, a system of references for people to find their way around in the environmental context [5].

Before these patients became ill with HIV infection, their perception of HIV

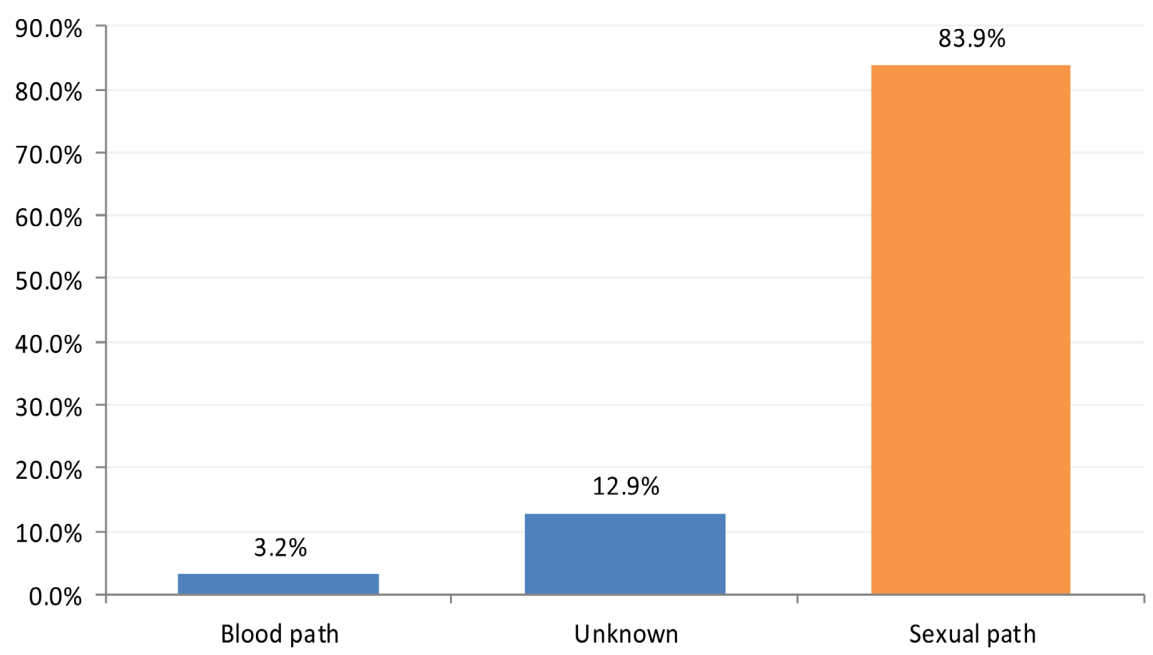

Figure 10. Distribution of mode of transmission among 31 PLHIV. 
infection was negative. HIV infection is seen as the disease of infidels, an incurable disease, a bad disease. The patients first clarified that Senegalese society has a negative perception of HIV infection and that they shared this same perception at some point, that is, before their illness. This meets the definition of social representation made by Jodelet (1994): "It is a form of knowledge, socially developed and shared, having a practical aim and contributing to the construction of a reality common to a social whole." [6] On the other hand, Moscovici specifies that by recognizing that representations are both generated and generating, one removes from them the pre-established, static side that they had in the classical vision [3]. This makes it possible to understand social and individual changes. So we can understand that our patients went from negative representations to positive representations in an average time of 11 years.

The study of social representations better corresponds to the needs of modern, changing and communicating society. Clenet (1998) specifies that "Social representations would be both interindividual, intergroup and ideological products and processes, which resonate with each other to form the dynamics specific to an institution and these dynamics are not indifferent as to the construction of individual representations" [4]. Thus the change of negative individual representations in Senegalese society must first pass through that of social representations. To say that a social representation has changed, it takes a change at the central nucleus level, otherwise, we are just talking about small changes. Denis M. explains that "The representations have an individual specificity but also a common nucleus shared by most human minds participating in the same culture" [7]. According to Abric (2003) the central core is a subset of the representation. It is made up of non-negotiable, stable and mutually consistent elements. The absence of any of these elements would deconstruct or give a radically different meaning to the representation as a whole [8]. Social representations are therefore susceptible to modification, but for this it is (in general) necessary that an element of the central nucleus be called into question. For the example of AIDS, one of the central elements is the mode of transmission of the disease. Also these elements of the central nucleus will determine the negative or positive nature of the representation. For example, sexual transmission was originally associated with marginalized groups, including sex workers, men who have sex with men, and drug users. This explains the first negative representations of HIV infection such as "gay cancer" in the United States in the 1980s [9]. The 25 out of 31 patients included in our study shared the same negative representations with those around them before becoming infected with HIV. Among these negative representations, the perception of HIV infection such as "the disease of infidels, vagrants" is the majority. The mode of transmission which is one of the elements of the central nucleus is the sexual route. He is associated with a marginal social group of infidels and vagrants. Which makes it a negative representation.

The image of HIV infection described as non-existent is considered negative. 
The patients concerned explain that despite the information heard through the media, they did not believe in the existence of the disease. This could be explained by the fact that these patients considered implausible the content of this information thus preventing the representation of the subject which here is HIV infection.

\subsection{Sharing of Positive HIV Status}

Sharing positive HIV status is difficult for 21 of the patients with fear of being stigmatized or discriminated against.

The main reason for the desired sharing of positive HIV status is based on a relationship of trust. The consequences of this voluntary disclosure are positive because either relationships with those around them remain unchanged or they tend to improve with a sustained presence of those around them. Women preferred to confide in their parents while men confided in their spouses. Only one woman confided in her husband because he insisted that she tell him the nature of his illness. Gruenais (1994) describes in his article the "storm in the homes" which would result from the information to the spouse and which must be avoided as well as the "heartbreak of families" [10]. For Gruenais, the spouse in the strict sense is not a parent and when informed, he is always likely to blame his partner for his infidelity. In addition, he specifies that the typical parent to be informed is the one who has the confidence of the patient and/or the practitioner [10].

Disclosure made independently of the patient's will is a source of stigma and discrimination with repercussions on psychological well-being. For one of our patients, the interpretation of the results of her analysis was made first by her friends with whom she shares the profession of sex workers. They all concluded that their friend's status was HIV positive because the medical team had not provided her with the results. So for these women receiving the envelope containing the results means "negative test" and the retention of the envelope means "positive test". Also two of our patients had their results received first by their supervisor. Thus it becomes necessary to improve sanitary devices for the respect of confidentiality.

\subsection{Stigma and Discrimination}

Moscovici (1961) testifies that representations make it possible to study social behavior and relationships without distorting or simplifying them [11]. Attitudes designate the evaluative side of representations [5]. Representations of HIV infection in Senegalese society are negative and this may explain attitudes such as discrimination and stigma.

HIV-related stigma and discrimination remain major obstacles to the effectiveness of the HIV response in all regions of the world. The non-disclosure of the seropositive status and the loss of follow-up after the announcement of seropositivity are major consequences. In fact, in our study, the main reason given 
that makes it difficult to share positive HIV status is fear of disclosure. In second place is the fear of being stigmatized.

Our study showed that 6 of the 31 PLHIV were victims of stigma such as rejection, avoidance, denigration, even leading to relocations. Of these 6 patients, 3 had current psychological repercussions such as depression or anxiety; two others voluntarily interrupted their treatment. According to Dupras (1990), the social stigmatization of people who are sick or in difficulty contributes to the deterioration of their mental health [12]. In the same vein, Sylla (2001) in her study concludes that taking into account psychological suffering among people living with HIV contributes to strengthening the support, support, listening and psychological care systems for people living with HIV [13]. So it becomes important to know the social perception of AIDS and to try to change it, if necessary [12].

\subsection{The Influence of the Media}

The modern world, in which information and communication play an essential role, offers the study of representations a new field. For Moscovici, the revolution brought about by mass communications, the dissemination of scientific and technical knowledge transforms ways of thinking and creates new content [14]. For 8 of our patients, Senegalese society's negative perception of HIV infection is due to early media announcements. They point out that the latter insisted on the origin of the affection which is frequently found in infidels and vagrants. They also accuse these media of insisting that the disease is fatal. Thus a policy based on communication for behavior change would cleanse the resistance noted in the fight against AIDS. This change must imperatively involve a reconstruction of representations about HIV infection.

\section{Conclusions}

Early representations of HIV infection from our patients are generally negative. They shared these same negative representations with those around them. The representations are dynamic. Negative representations have a negative impact on adherence to ARV treatment and on the patient's psychological state. Socially, they are the source of negative attitudes such as discrimination and stigmatization. Sharing positive HIV status when you want it and to the person the patient trusts only has positive results. Disclosure of positive HIV status, regardless of the patient's wishes, is a source of discrimination and stigmatization as those around them have negative representations of HIV infection. It often causes psychological suffering. The media influence representations but information or communication alone cannot eliminate the discrimination faced by PLHIV.

In our context, the eradication of discrimination among PLHIV must imperatively involve a reconstruction of individual, collective and social representations. And to achieve it, it will require collaboration with specialists in Psychism. This will make it possible to obtain, in addition to effective and efficient results, a real and lasting impact of all the efforts made in the fight against HIV. 


\section{Conflicts of Interest}

The authors have no conflict of interest.

\section{References}

[1] Cissé, V.M.P. (2013) L'infection à VIH: Connaissances, attitudes et pratiques des personnes vivant avec le VIH suivies au centre de recherche clinique et de formation du CHNU de Fann. Thèse Doctorat Médecine, Université Cheikh Anta Diop, Dakar, numéro 144, 103.

[2] Silvestre, D., Linard, F. and Beau, P. (1995) Psychiatrie et infection à VIH chez l'adulte. Elsevier, Paris, 25.

[3] Moscovici, S. (1989) Des représentations collectives aux représentations sociales. In: Jodelet, D., Ed., Les représentations sociales, PUF, Paris, 63.

[4] Clenet, J. (1998) Représentations, formation et alternance. Harmattan, Paris, 70.

[5] Benabdelmalek, A. (2005) Les représentations sociales des enseignants du collège d'enseignement moyen en situation de violences. Mémoire de Magistère en Psychologie sociale. Faculté des sciences sociales et humaines, Département de psychologie et sciences de l'éducation. Université Mentouri de Constantine, Constantine, 184.

[6] Jodelet, D. (1994) Les représentations sociales, regard sur la connaissance ordinaire. PUF, Paris, 36-57.

[7] Denis, M. (1989) Image et cognition. PUF, Paris, 33.

[8] Abric, J-C. (2003) Pratiques sociales et représentations. Presses Universitaires de France, Paris, 11-36. https://doi.org/10.3917/eres.abric.2003.02.0011

[9] Grmek, M.D. (1989) Histoire du sida. Payot, Paris, 15-29.

[10] Gruenais, M.E. (1994) Qui informer au Congo: Malades, familles, tradipraticiens, religieux? Psychopathologie africaine, XXVI, 189-209.

[11] Moscovici, S. (1961) La psychanalyse, son image et son public. Thèse principale de Doctorat ès letters, P.U.F, Paris, 652.

[12] Dupras, A. (1992) Dossier Sida et Santé mentale. Santé mentale au Québec, XVII, 10. https://doi.org/10.7202/502053ar

[13] Sylla, O., Boissy, L. and Bronsard, G. (2001) Exploration de l'état psychologique des patients vivant avec le VIIH/SIDA inclus dans des protocoles de multithérapie antirétrovirale à Dakar. Dakar.

[14] Aimon, D. (2015) Concept de représentation. http://daimon.free.fr/mediactrices/representations.html 